\title{
Primary Subdural Extramedullary Neurocytoma: A Case Report
}

\author{
Sihua Yang, Jiawei Yang, Jian Hu and Guangyu Tang* \\ Department of Radiology, Tenth People's hospital of Tongji, University School of Medicine, China
}

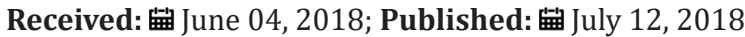

*Corresponding author: Guangyu Tang, Chief Doctor and Professor, Department of Radiology, Shanghai Tenth People's Hospital of Tongji University School of Medicine, China

\begin{abstract}
Neurocytoma as an extramedullary lesion is extremely rare in spinal canal. We present a case with primary subdural extramedullary neurocytoma of thoracic-lumbosacral spine. The patient presented with sore waist and numbness of right foot for half a month, as well as progressively increasing weakness of both lower limbs. An extramedullary tumor was revealed in the thoracic-lumbosacral level bymagnetic resonance imaging(MRI) and confirmed by pathology as extra ventricular Neurocytoma. In conclude, whenever a patient is diagnosed to have an extramedullary tumour in the thoracic-lumbosacral level, neurocytoma should be considered within the differential diagnosis despite its rarity.
\end{abstract}

\section{Case Presentation}

A 61-year-old male was referred to our hospital in September 2017, complaining sore waist associated with numbness of right foot, as well as progressively increasing weakness of both lower limbs for half a month. On examination, the patient presented with positive straight leg raising sign, increased deep tendon reflexes, and muscle strength in double lambs was rated as grade $3 / 4$. The initial laboratory results were normal. MR images showed an extremely extensive extramedullary subdural lesion, which extended from T10 vertebral level to the S2 vertebral level. The spinal cord was normal and the tumor had a well-defined plane of cleavage with it. The tumor showed isointense signal on T1weighted images (Figure 1A), slightly hyperintense signal intensity on T2-weighted images (Figures 1B\&1D) and a hyperintense signal intensity on diffusion weighted images(DWI) (Figure 1C). It presented avidly heterogeneous enhancement on T1-weighted images after gadolinium administration (Figure 2) which clearly emphasized that the tumor was extramedullary. An operation was performed through T12 to L4 laminotomy. The tumor showed rich hypervascularity appearance and a clear boundary with nearby spinal cord on surgery. Because the lesion was too extensive to be entirely removed, a partial resection was performed. When discharged, the patient's the neurological status was improved with negative straight leg raising sign, and muscle strength in double lambs was rated as grate 2/3. Microscopically, the tumor was made of sheets of small round or oval cells, and most of cells presented clear perinuclear halos. A cell-free zone composed of subtle fibrillary neuropils was found. The phenomenon such as calcification, mitosis, necrosis within lesion was not observed (Figure 3).
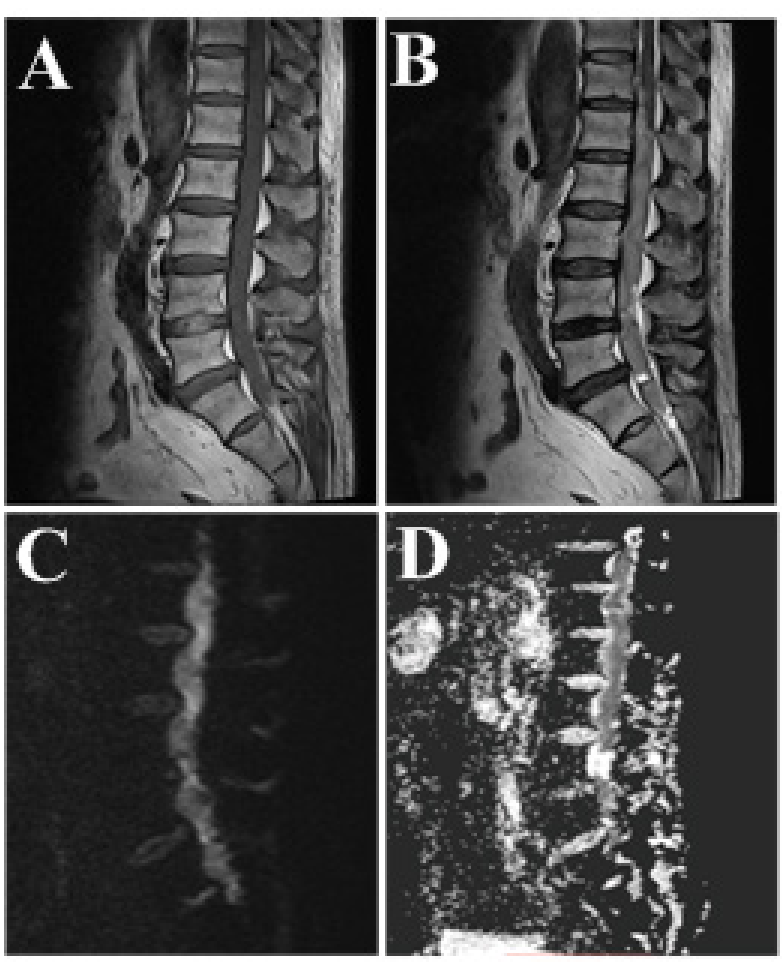

Figure 1: Magnetic resonance image of the lumbar spine. A mass in thoracio-lumbosacral spine shows an isointense on. sagittal T1W image (A) and slightly hyperintense signal on T2W image (B). It shows a hyperintense signal on DW image $(\mathrm{C})$ and corresponding decreased ADC value (D). 


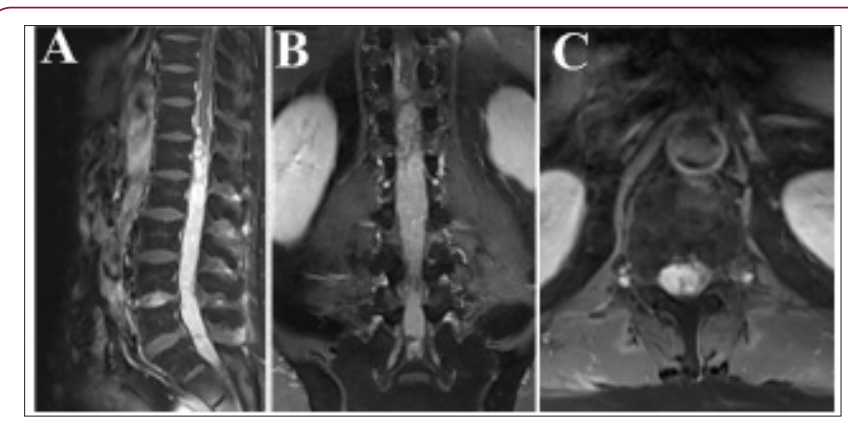

Figure 2: The tumor showed avidly heterogeneous enhancement on T1W images after gadolinium administration.

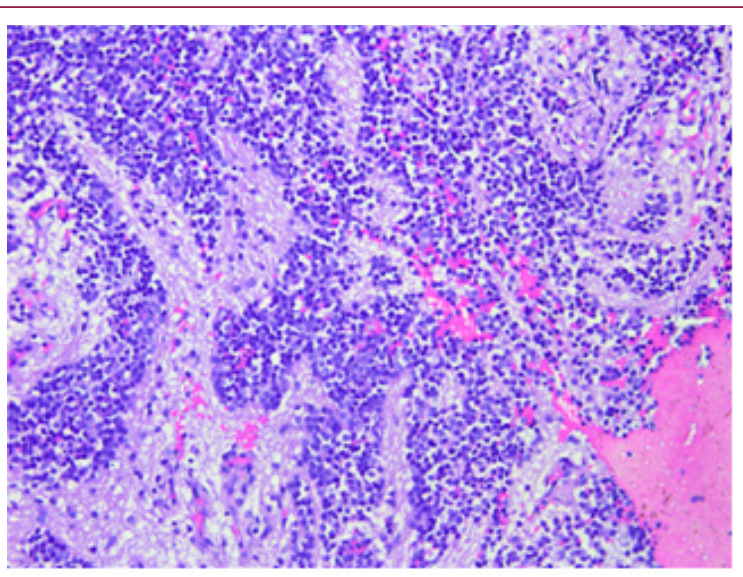

Figure 3: Histological observation. The tumor was made up with sheets of small round or oval cells (arrow), and most of them presented clear perinuclear halos. A cell-free zone composed of subtle fibrillary neuropils(triangle) was showed.

\section{Discussion}

Findings of Extramedullary neurocytoma in thoraciolumbosacral spine have been reported up to now. An issue of significant importance is that spinal neurocytomas have not specific imaging features and can be easily misleading. On MRI, neurocytoma usually present hypointense or isointense signal on T1W images and isointense or hyperintense signal on T2W images [1]. Decreased ADC value is perhaps characteristic for EVN, which is in accordance with the pathological findings that the tumor was made up with sheets of small round or oval cells. After gadolinium administration, the lesions usually displayed heterogenous enhancement. It is related to the tumor structure which is composed of both sheets of small round cells and cell-free zone composed of subtle fibrillary neuropils. It is extraordinary difficult to make a definitive diagnosis of neurocytomas only based on MRI. The final diagnosis depends on histopathological examination. The differential diagnosis of spinal neurocytoma, just like supratentorial CN, typically includes ependymoma and oligodendroglioma[2].

The optimal treatment is gross total resection for spinal neurocytoma. If it is too extensive to be excised, Central Neurocytoma $(\mathrm{CN})$ is a rare tumor that is typically located in the supratentorial ventricular system near the When neurocytoma occur outside its typical sites, it is named as extraventricular neurocytoma (EVN). So far, EVNs was founded in many locations, such as cerebral parenchyma, thalamus, cerebellum. However, EVN occured in the spinal canal is extremely rare and is always found in intramedullary locations [3]. In our case, the most striking feature of the tumor is that it grows in the spinal canal as intradural extramedullary tumor, which is extremely rare and uncommon. To our best knowledge, no report with respect to imaging a partial resection is also advocated and effective. In addition to that, long-term follow-up is suggested due to the potentiality for recurrence. EVN occurred in the spinal canal is extremely rare. Our case suggests that it should be considered in presurgical differential diagnosis for intradural extramedullary tumors.

\section{References}

1. Polli FM, Salvati M, Miscusi M, Delfini R, Giangaspero F (2009) Neurocytoma of the spinal cord: report of three cases and review of the literature. Acta Neurochirurgica 151(6): 569-574.

2. Sharma S, Sarkar C, Gaikwad S, Suri A, Sharma MC (2005) Primary neurocytoma of the spinal cord: a case report and review of literature. Journal of Neuro-Oncology 74(1): 47-52.

3. Hartmann DP, Azumi N, Ozdemirli M, Makuria AT, Rushing EJ, et al. (2007) Oligodendroglioma with neurocytic differentiation versus atypical extraventricular neurocytoma: a case report of unusual pathologic findings of a spinal cord tumor. J Neu-rooncol 82(2): 199 205.

\section{ISSN: 2574-1241}

\section{DOI: 10.26717/BJSTR.2018.06.001394}

Guangyu Tang. Biomed J Sci \& Tech Res

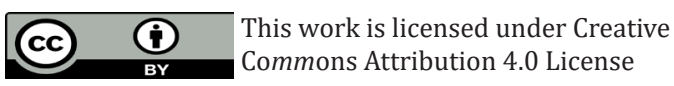

Submission Link: https://biomedres.us/submit-manuscript.php

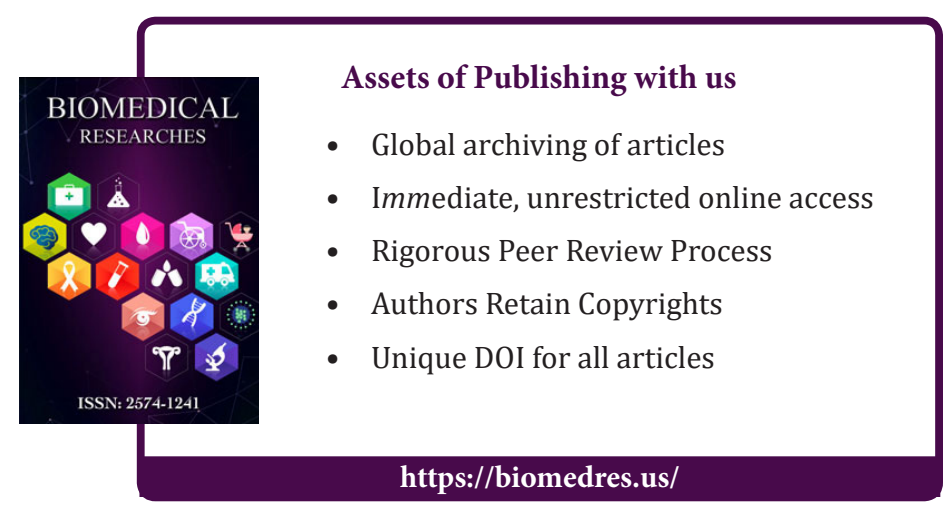

